\title{
THE CONVECTION TERM EVALUATION \\ OF THE TURBULENCE KINETIC ENERGY BALANCE IN A FLAME WITH MEDIUM DENSITY CHANGES
}

\author{
Grzegorz, Grodzki \\ Institute of Computer and Information Sciences \\ Czestochowa University of Technology, Poland \\ grzegorz.grodzki@icis.pcz.pl
}

\begin{abstract}
This paper consists of an analysis pertaining to the turbulence kinetic energy balance equation with the acknowledgement of burning medium density change influence. The evaluation of the convection element value and of the density change influence on the turbulence kinetic energy balance has been attempted. Experimental data obtained by means of laser Doppler anemometry and the radial distributions of average temperatures of a burning jet have been exploited in the present paper.
\end{abstract}

\section{Introduction}

The aerodynamic characteristics of flows with combustion, which covered average and fluctuating velocity components associated with a thermal field, have been presented in conducted tests [1]. Presented data allows one to assess the relationship between the flame type, the location of the combustion front and the particular components of the turbulence kinetic energy balance by revealing flow turbulence characteristics with combustion.

It has been stated that, in particular, both generation and dissipation of the turbulence kinetic energy is concentrated in the inner range of the burning jet limited by the combustion front surface. These processes do not equilibrate each other in any considered cross-section, which proves that remaining components thereof (convection and diffusion) have an essential role in closing the energy balance.

The results presented in the work [1] pertain to a free axisymmetric burning jet produced as a result of combustion of a mixture of methane with air and a comparative cold jet. Kinetic and kinetic-diffusion flame group has been covered by the primary studies. In order to differentiate their structures various mixture ratios of methane with air have been used, where the volume concentration has been changing within $11 \div 50 \%$. This composition of the combustible mixture corresponded to the air excess factor values within the scope from $\lambda=0.85$ (close to kinetic flame) to $\lambda=0.11$. Jet velocity at the nozzle exit $\overline{U_{o}}$ has been changed in the range 
$(6.67 \div 7.17 \mathrm{~m} / \mathrm{s})$ in order to maintain a constant Reynolds number in the presence of a various combustible mixture composition.

Reynolds number defined by rates characterizing the conditions at the nozzle exit $d=8 \mathrm{~mm}$ in diameter has been maintained at $\mathrm{Re}_{\mathrm{d}}=3650$, that guaranteed the turbulent character of the flow.

The velocity field measurements of the analysed flow was conducted in the range of $\mathrm{x} / \mathrm{d}=4 \div 38$, where particular measurement cross-sections were spaced with the value of $\Delta \mathrm{x}=2 \mathrm{~d}$. The radial range of the measurement zone was $\mathrm{r} / \mathrm{d}=0 \div 4$.

On the basis of the conducted tests an attempt to define the components of the turbulence kinetic energy balance has been made, where the medium density changes in the burning jet range were omitted in order to facilitate the process. In reality, however, strong temperature gradients occur in the range of the flame, thus strong medium density changes are also present. The turbulent character of the flow also leads to the occurrence of temperature fluctuation, which causes density fluctuation. The composition of the combustible mixture also changes, thus the change of the flowing mixture density is also dependent on the composition of combustion products which change in the jet range. The described mechanisms imply that the previous method defining the turbulence kinetic energy balance must be verified, and they particularly impose the necessity to consider medium average density changes.

\section{The turbulence kinetic energy transport equation in a free round jet}

The energy equation [2] for the axisymmetric jet in a non-dimensional form is presented by the relation

$$
\begin{aligned}
& \underbrace{\frac{1}{\rho} \frac{d}{\bar{U}_{o x}^{3}}\left[\overline{u_{x} \frac{\partial}{\partial x} p}+\overline{u_{r} \frac{\partial}{\partial r} p}\right]}_{1}+\underbrace{\frac{d}{\bar{U}_{o x}^{3}}\left[\overline{u_{x} \frac{\partial}{\partial x} \frac{q^{2}}{2}}+\overline{u_{r} \frac{\partial}{\partial r} \frac{q^{2}}{2}}\right]}_{2}+\underbrace{\frac{d}{\bar{U}_{o x}^{3}}\left[\overline{U_{x} \frac{\partial}{\partial x} \frac{q^{2}}{2}}+\overline{U_{r}} \frac{\partial}{\partial x} \overline{\frac{q^{2}}{2}}\right]}_{3}+ \\
& +\underbrace{\frac{d}{\bar{U}_{o x}^{3}}\left[\overline{u_{x}^{2}} \frac{\partial \overline{U_{x}}}{\partial x}+\overline{u_{x} u_{r}}\left(\frac{\partial \overline{U_{x}}}{\partial r}+\frac{\partial \overline{U_{r}}}{\partial x}\right)+\overline{u_{r}^{2}} \frac{\partial \overline{U_{r}}}{\partial r}\right]}_{4}+ \\
& +\underbrace{v \frac{d}{\bar{U}_{o x}^{3}}\left\{\frac{\partial^{2} \overline{q^{2}}}{\partial x^{2}}+\frac{\partial^{2} \overline{q^{2}}}{\partial r^{2}}+\frac{\partial}{\partial x}\left[\frac{\partial}{\partial x}\left(\overline{u_{x} u_{x}}\right)+\frac{1}{r} \frac{\partial}{\partial r}\left(r \overline{u_{x} u_{r}}\right)\right]+\frac{\partial}{\partial r}\left[\frac{\partial}{\partial x}\left(\overline{u_{x} u_{r}}\right)+\frac{1}{r} \frac{\partial}{\partial r}\left(r \overline{u_{r} u_{r}}\right)\right]\right\}}_{5}+\underbrace{\frac{d}{\bar{U}_{o x}^{3}}}_{6} \in=0
\end{aligned}
$$

in which kinetic energy has been formulated in the form:

$$
\overline{q^{2}}=\frac{1}{2}\left(\overline{u_{x}^{2}}+\overline{u_{r}^{2}}\right)
$$


The particular terms mean: (1) pressure diffusion, (2) turbulence kinetic energy diffusion, (3) energy convection, (4) the production of turbulence kinetic energy, (5) viscous stress work, and (6) the dissipation of energy into heat.

The turbulence kinetic energy transport is carried out by the following processes:

- convection (term 1) captivating its stream which is lifted by the average movement,

- work executed by the turbulent pressure component (term 2) and stresses in the random movement caused by liquid viscosity (term 5),

- diffusion carried out by random movement (term 3), production referring to the energy stream deriving from the average movement (term 4),

- dissipation (term 6) presenting the turbulence energy convection into the inner medium energy.

The equation analysis (1) indicates that the assigned form pertains to the incompressible flow, because it does not include the medium density change. The above-mentioned burning jet subjected to the analysis, which was formed as a result of methane with air mixture combustion, is characterized by a strong density change.

An attempt to verify the established turbulence kinetic energy balance equation has been made in the present work because the acknowledgement of information on the conversion dynamics of the turbulence kinetic energy may provide very valuable experimental data, which may constitute the basis for the verification concerning the aerodynamic model of combustion.

\section{The rules of averaging with the observance of density changes}

In accordance with the Reynolds hypothesis, the instantaneous value of the physical quantity characterizing the turbulent flow may be regarded as an aggregate (averaged in time) of average and fluctuant values.

$$
\begin{aligned}
U_{i} & =\overline{U_{i}}+u_{i} \\
\rho & =\bar{\rho}+\widetilde{\rho} \\
P & =\bar{P}+p \\
\Theta & =\bar{\Theta}+v
\end{aligned}
$$

After the implementation of the above relations into the continuity and momentum equations with the observance of the averaging in time procedure it converts them in the following form:

$$
\frac{\partial \bar{\rho}}{\partial t}+\frac{\partial}{\partial x_{i}}\left(\overline{\rho U_{i}}+\overline{\tilde{\rho} u_{i}}\right)=0
$$




$$
\begin{aligned}
& \frac{\partial}{\partial t}\left(\bar{\rho} \bar{U}_{i}\right)+\frac{\partial}{\partial x_{j}}\left(\bar{\rho} \bar{U}_{i} \bar{U}_{j}\right)+\frac{\partial}{\partial t}(\tilde{\rho} U)+\frac{\partial}{\partial x_{j}}\left(\bar{\rho} \bar{u}_{i} u_{j}+\overline{\tilde{\rho} u_{i}} \bar{U}_{j}+\overline{\tilde{\rho} u_{j}} \bar{U}_{i}+\overline{\tilde{\rho}} \overline{u_{i} u_{j}}\right)= \\
& =\bar{F}_{i}-\frac{\partial \bar{P}}{\partial x_{i}}+\frac{\partial}{\partial x_{j}}\left[\mu\left(\frac{\partial \bar{U}_{i}}{\partial x_{j}}+\frac{\partial \bar{U} j}{\partial x_{i}}\right)\right]-\frac{2}{3} \frac{\partial}{\partial x_{i}}(\mu \bar{\Theta})
\end{aligned}
$$

After the observance of the Reynolds hypothesis, additional components: $\left(\overline{\tilde{\rho} u_{i}} ; \overline{u_{i} u_{j}}\right)$ and $\left(\overline{\tilde{\rho} u_{i} u_{j}}\right)$ have occurred in the above equations, which present correlations between velocity and density fluctuating components. These correlations are the minor functions of dimension and time coordinates.

Should the conventional averaging time procedure be substituted by the density weighted mean, used in the work [3] and developed in [4] and [5], the density average velocity rate $\bar{U}_{i}^{o}$ results from the defining relation:

$$
\bar{\rho} \bar{U}_{i}^{o}=\overline{\rho U}_{i}
$$

where

$$
\bar{U}_{i}^{o}=\frac{\overline{\rho U}_{i}}{\rho}
$$

instantaneous velocity may be presented as follows:

$$
U_{i}=\bar{U}_{i}^{o}+u_{i}^{o}=\bar{U}_{i}+u_{i}
$$

The instantaneous velocity of the intersection $\rho U_{i}$ :

$$
\rho U_{i}=(\bar{\rho}+\tilde{\rho})\left(\bar{U}_{i}^{o}+u_{i}^{o}\right)=\bar{\rho} \bar{U}_{i}^{o}+\tilde{\rho} \bar{U}_{i}^{o}+\rho u_{i}^{o}
$$

after the averaging in time the expression is equal to zero

$$
\overline{\tilde{\rho} \bar{U}_{i}^{o}}=\overline{\tilde{\rho}} \bar{U}_{i}^{o}=0
$$

may be formulated in the following form:

$$
\overline{\rho U}_{i}=\bar{\rho} \bar{U}_{i}^{o}+\overline{\rho u_{i}^{o}}
$$

The comparison to the relations (6) creates the possibility to formulate a notation:

$$
\overline{\rho u_{i}^{o}}=\overline{(\bar{\rho}+\tilde{\rho}) u_{i}^{o}}=0
$$


where the dependence is:

and

$$
\begin{gathered}
\overline{\tilde{\rho} u_{i}^{o}}=-\overline{p u_{i}^{o}} \\
\overline{u_{i}^{o}}=\frac{-\overline{\tilde{\rho} u_{i}^{o}}}{\bar{\rho}}
\end{gathered}
$$

It indicates the basic difference between both averaging methods considered in this paper, which may be summarized by the following arrangement:

$$
\begin{array}{lll}
\text { conventional averaging: } & \overline{u_{i}}=0 ; & \overline{\rho u_{i}}=\overline{\tilde{\rho} u_{i}} \neq 0 ; \\
\text { density-weighted averaging: } & \overline{u_{i}}=0 ; & \overline{\rho u_{i}}=\overline{\tilde{\rho} u_{i}} \neq 0 ;
\end{array}
$$

The relations between average velocities result from the equation (7), which after averaging may be formulated as follows:

$$
\overline{u_{i}^{o}}=\bar{U}_{i}-\bar{U}_{i}^{o}
$$

Because conventional average

$$
\overline{\rho U}_{i}=\bar{\rho}_{i}+\overline{\tilde{\rho} u_{i}}=\bar{\rho} \bar{U}_{i}^{o}
$$

thus

$$
\overline{u_{i}^{o}}=\bar{U}_{i}-\bar{U}_{i}^{o}=-\frac{\overline{\tilde{\rho} u_{i}}}{\bar{\rho}}
$$

that, after the comparison to (9), entitles to formulate a notation:

$$
\overline{\tilde{\rho} u_{i}}=\overline{\tilde{\rho} u_{i}^{o}}
$$

The dependency (7) combines the density and velocity covariance, determined with respect to both considered averaging procedures.

The described density averaging procedure reduces the continuity equation and momentum conservation in a significant manner, eliminating the covariance $\overline{\widetilde{\rho} u_{i}}$ of the first equation, whereas $\overline{\widetilde{\rho} u_{i}}$ and $\overline{\widetilde{\rho} u_{j}}$ from the second equation. Covariances eliminated in this manner have significant values in the case of compressible flows, thus they cannot be omitted.

\section{The temperature field analysis of the burning kinetic-diffusion jet}

The velocity field structure in the flow with combustion remains in a tight relation with temperature variability, which leads to the diversification of medium density in the flame. The presented results reveal that the development of radial temperature distributions is affected by the fuel-air mixture composition which characterizes the group of kinetic-diffusion flames subjected to testing. 


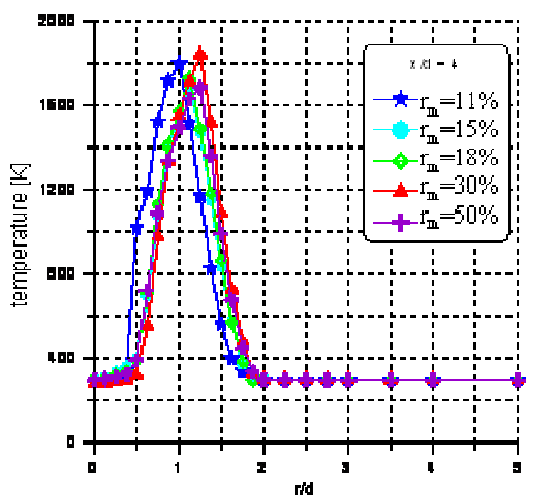

Fig. 1. The average temperature radial profiles of the flame group subjected to testing at a distance of $\mathrm{x} / \mathrm{d}=4$

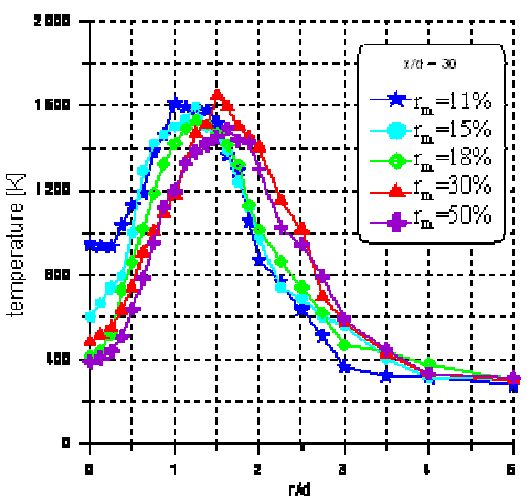

Fig. 2. The average temperature radial profiles of the flame group subjected to testing at a distance of $\mathrm{x} / \mathrm{d}=30$

Data depicted in Figures 1 and 2 indicate that as the distance from the nozzle exit becomes grater then the average temperature radial profiles are subjected to evolution, which is characterized by, inter alia, the higher temperature zone extension with simultaneous distance growth from the flame axis. It should be emphasized that the temperature in the middle section of the flame maintains a relatively low level at a great distance from the nozzle exit. It pertains to the whole flame group subjected to the analysis. Differences resulting from the volume changes of the mixture composition are difficult to apprehend while the methods of data presentation in Figures 1 and 2 are observed. They are, however, more visible in the following graph, in which the temperature field heterogeneity measure in a flame has been defined by its maximal difference in the flame cross-section $\Delta T_{\max }=T_{\max }-T_{c}$.

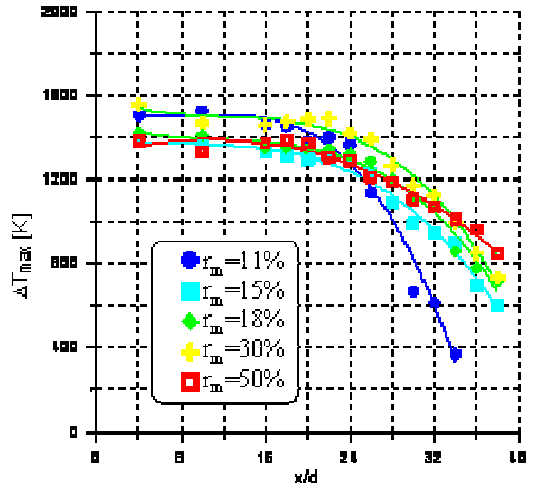

Fig. 3. Maximal temperature differences in the flame cross-section with a variant mixture composition

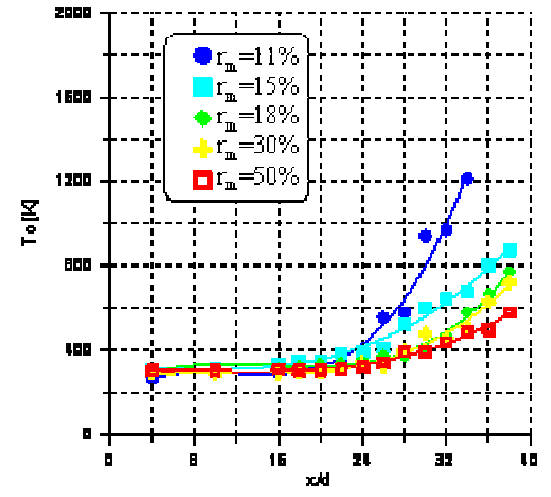

Fig. 4. Temperature evolution in the burning jet axis for the kinetic-diffusion flame group subjected to testing 
The change pattern of this rate in the direction of the flow is depicted in Figure 3 , whose supplementation is Figure 4 that presents the temperature evolution in the burning jet axis. Applied patterns in Figures 3 and 4 pertain to the various volume values of the fuel portion in the combustible mixture. Differences between the maximal temperature in a given cross-section of the burning jet and its axis temperature $\Delta T_{\max }=T_{\max }-T_{c}$ start to decline not until the distance is greater $\mathrm{x} / \mathrm{d} \geq 22$ (Fig. 3). Temperature field equalization in the inner range of the flame occurs mainly by its growth in the flame axis, and the observed changes begin to be more visible in flames with a lower fuel portion in the combustible mixture (Fig. 4).

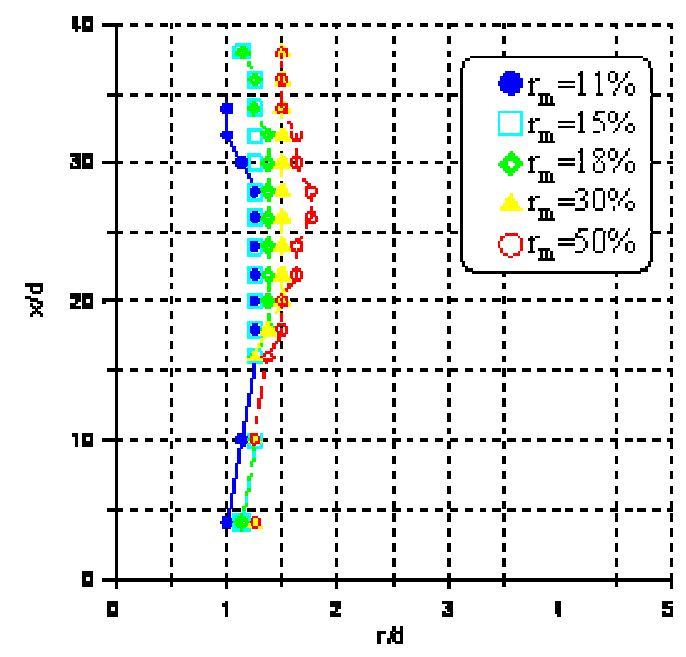

Fig. 5. The influence of the combustible mixture composition on the maximal temperature lines in burning jets

Average flame front position, described by the radial coordinate value $(\mathrm{r} / \mathrm{d})_{\mathrm{T}=\mathrm{T} \max }$, where the temperature reaches its maximal level, is important information that results from the profile shape analysed herein. Maximal temperature lines, indicating the average position of the reaction zone within the flames subjected to testing are presented in Figure 5. Applied results indicate the increase of the values $(\mathrm{r} / \mathrm{d})_{\mathrm{T}=\mathrm{Tmax}}$ in the initial range of the flame, and then they stabilize on the levels dependent upon the fuel-air mixture composition. The line corresponding to the kinetic flame type $\left(r_{m}=11 \%\right)$ is closest to the flame axis.

The inner range of the burning jet contained within the limits set by the combustion front line is characterized by strong radial temperature gradients. Therefore, the increased stream of the volume factor is concentrated in this region. A zone, in which a low temperature level is maintained, is positioned along the flame axis. 


\section{The influence of temperature on the turbulence kinetic energy balance structure on the example of the convection term}

The cited description of the turbulence kinetic energy transport processes in free burning round jets was based on the analysis of the energy stream distribution variation on the example of the convection element in the flow range subjected to testing. The radial distribution of the analysed values was determined by the experimental data collected in the following control cross-sections of the jet.

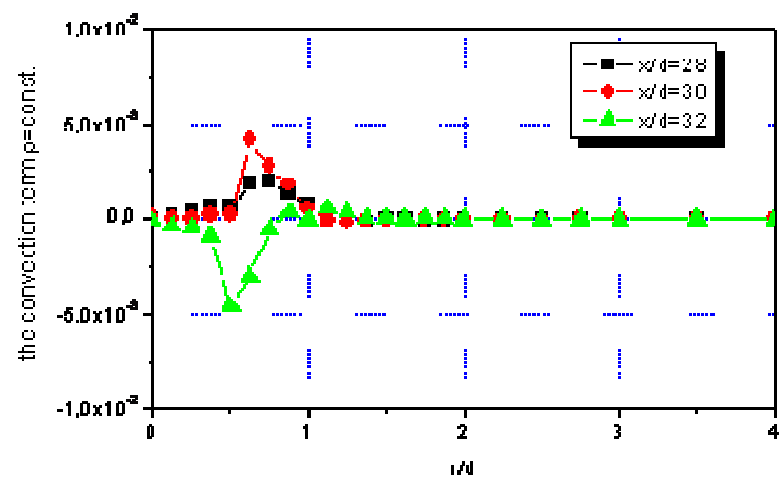

Fig. 6. The evolution of the convection term in the flame with large fuel portion $r_{m}=50 \%$ in the combustible mixture without the observance of density changes

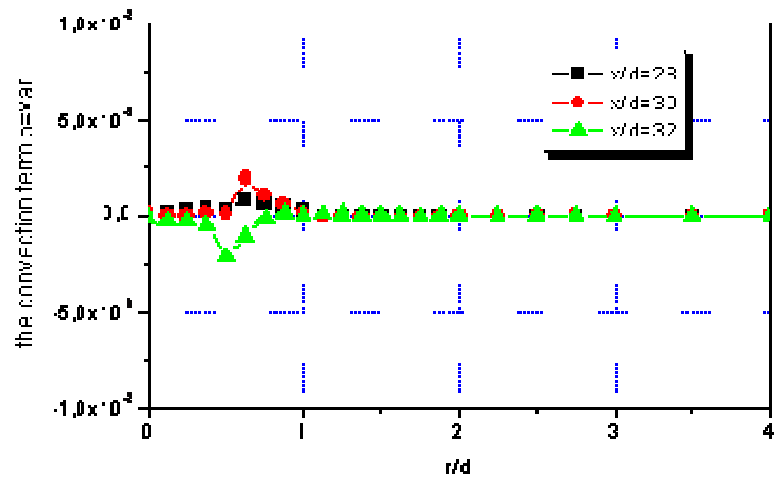

Fig. 7. The evolution of the convection term in the flame with large fuel portion $r_{m}=50 \%$ in the combustible mixture with the observance of density changes

In order to define the stream values of the turbulence kinetic energy transferred by means of convection it was necessary to determine the dimensional derivatives of the chosen flow parameters in the longitudinal and radial direction. These derivatives were determined by the numerical analysis by means of the experimental values from adjacent test points. 
The term (1) of the energy balance equation (1) presents the element responsible for the convection of the turbulence kinetic energy. The analysis of the term component (1) results from the necessity to simultaneously use the dimensional differentiation in the longitudinal and radial direction.

During the determination of its values object files, which were made from the digital measurement signal processing recorded by the laser Doppler anemometry, have been the basis for calculations. Data recorded in each set in the form of records include the values: $\bar{U}_{x}, \bar{U}_{r}, \overline{u_{x}^{2}}, \overline{u_{r}^{2}}, \overline{u_{x} u_{r}}$, respectively, in individual test points. Sets prepared in this manner constituted the basis for the calculations of the developed numerical computational algorithm of the convection element.

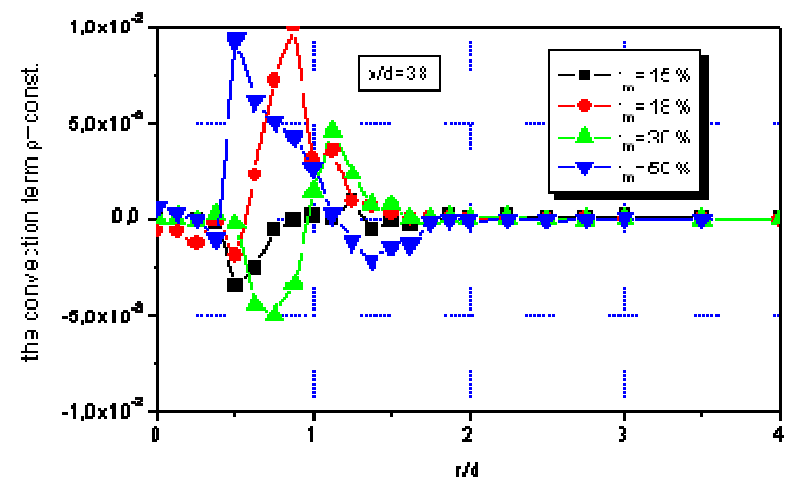

Fig. 8. The convection term change course for the analysed flame group without the observance of density changes

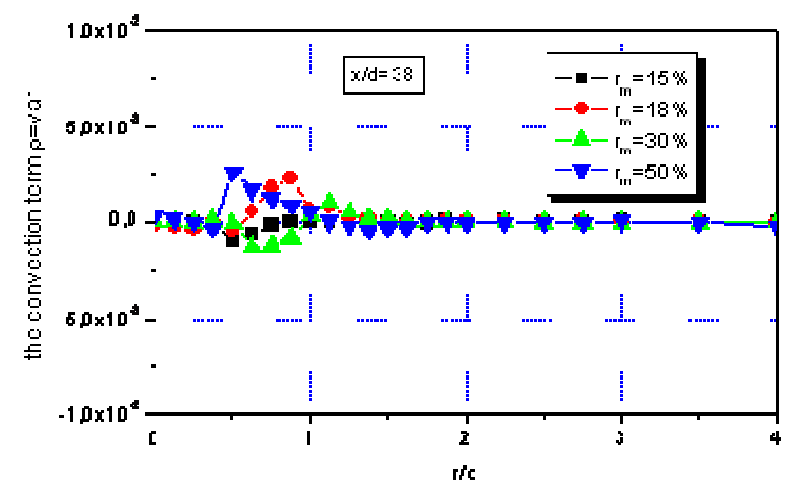

Fig. 9. The convection term change course for the analysed flame group with the observance of density changes

The comparative analysis (Figs 6 and 7), depicting the evolution of the convection element in the flame $\mathrm{r}_{\mathrm{m}}=50 \%$ for the case pertaining to the constant density (Fig. 6) and with the observance of the medium density change (Fig. 7), indicates 
that in all control cross-sections the qualitative changes of the energy distributions transferred by means of convection are not visible. A similar conclusion may be made on the basis of Figure 8, in which analogical distributions set at a distance of $\mathrm{x} / \mathrm{d}=38$ for chosen kinetic-diffusion flames have been presented.

The analysis of the consecutive Figure 9 indicates that in the case of the convection element with a constant density the energy values determined in the hightemperature zone in the combustion front region are approximately five times higher than in the case when the changes $\rho$ are observed.

\section{Conclusions}

Due to the fact that the acknowledgement of information pertaining to the change dynamics of turbulence kinetic energy may provide very valuable experimental data, which may constitute the basis for the verification of the aerodynamic model of combustion, an attempt was made in this work to verify the turbulence kinetic energy balance equation.

In order to define the values of the turbulence kinetic energy streams by means of convection it was necessary to determine the dimensional derivatives of chosen flow parameters in the longitudinal and radial direction. These derivatives were determined by the numerical method by using the experimental values obtained from the adjacent test points.

The analysis of the energy balance equation, especially of the element components responsible for the turbulence kinetic energy convection, indicates that the simultaneous implementation of dimensional differentiation in the longitudinal and radial direction is necessary.

In summary, it may be stated that the observance of temperature changes does not change the qualitative character of the equation elements of the flow kinetic energy balance, it may, however, change the relations between individual equation elements.

\section{References}

[1] Grodzki G., The turbulence structure of the free kinetic-diffusion flames - doctoral dissertation, Institute of Thermal Machinery, Czestochowa University of Technology 2000.

[2] Elsner J.W., Flow Turbulence, PWN, Warsaw 1987.

[3] Van Driest E.R., Turbulent boundary layer in compressible fluids, J. Aeronaut. Sci. 1951, $18,145$.

[4] Favre A., Statistical equation of turbulent gases, SIAM Problems of Hydrodynamics and Continuum Mechanics, J. Mec. 1965, 4, 361.

[5] Cebeci T., Smith A.M.O., Analysis of Turbulent Boundary Layers, Academic Press, New York 1974. 